\title{
Les techniques d'information, de communication, de documentation et l'enseignement
}

\section{Emile Gaspari}

\section{Q OpenEdition}

\section{Journals}

Édition électronique

URL : http://journals.openedition.org/trema/2425

DOI : $10.4000 /$ trema.2425

ISSN : 2107-0997

Éditeur

Faculté d'Éducation de l'université de Montpellier

\section{Édition imprimée}

Date de publication : 1 mai 1992

Pagination : 17-30

ISSN : 1167-315X

\section{Référence électronique}

Emile Gaspari, « Les techniques d'information, de communication, de documentation et

l'enseignement », Tréma [En ligne], 1 | 1992, mis en ligne le 02 décembre 2013, consulté le 10

décembre 2020. URL : http://journals.openedition.org/trema/2425; DOI : https://doi.org/10.4000/

trema. 2425

Ce document a été généré automatiquement le 10 décembre 2020.

Trema 


\title{
Les techniques d'information, de communication, de documentation et l'enseignement
}

\author{
Emile Gaspari
}

\section{Bref historique}

\section{Une histoire chaotique}

1 L'introduction des nouvelles technologies, informatique et audiovisuel, a relevé de trois types d'objectifs :

- sensibilisation aux incidences sociales et culturelles du développement de l'audiovisuel et de l'informatique,

- étude des outils et formation à l'usage,

- utilisation des technologies à des fins pédagogiques.

- Ces objectifs sont en fait complémentaires, mais l'histoire mettra l'accent sur l'un ou l'autre...

\section{L'audiovisuel, une ancienne nouvelle technologie}

2 L'image a eu une fonction pédagogique dès que sa reproduction a été possible. Elle a progressivement gagné en attrait : couleur, photographie. La diapositive, le film $(16 \mathrm{~mm}$ et $8 \mathrm{~mm}$ ), sont encore en service, bien que la production ait cessé depuis quelques années (les produits ont en général vieilli, et ne correspondent plus aux exigences de qualité de notre époque). Après la période faste des années 60 et du début des années 70, de la radiotélévision scolaire, - rappelons à ce propos que la France a été un pionnier de l'enseignement à distance et de la radiotélévision scolaire -, l'allègement et la baisse des coûts des matériels, le magnétoscope et son complément, la caméra, ouvraient des perspectives: le montage facile, la possibilité pour l'enseignant de créer ses propres documents laissaient envisager une production de masse et des échanges. Les Ecoles 
Normales en étaient équipées dès le début des années 70, en même temps que se créaient les circuits fermés, pour l'enregistrement de classes et l'autoscopie. Le comité de coordination des Ecoles Normales animait un réseau d'échanges. Désillusion : le CCEN est dissous en fin des années 70 sans que les travaux en cours aient pu donner matière à des produits portables et enrichissables.

3 L'expérience «Jeune Téléspectateur Actif» fera l'objet d'un pilotage conjoint de l'Education Nationale et de la Jeunesse et des Sports, son objet sera d'analyser avec les enfants le message audiovisuel. Elle sera abandonnée sans évaluation, et surtout, cette fois encore, avant que les actions de terrain n'aient pu donner lieu à une publication élaborée.

4 Dans la première moitié des années 80 , le plan vidéo-collèges, équipant des collèges en magnétoscopes et caméras vidéos, montrera l'intérêt des élèves pour leur participation à une expression utilisant les moyens audiovisuels. A l'inverse l'opportunité des équipements en laboratoires de langues reste controversée.

\section{L'informatique, essais réussis et transformations manquées}

5 L'informatique va nourrir des espoirs encore plus démesurés. Dès que les ordinateurs deviennent accessibles, la France se lance dans l'expérience des minis dans 58 lycées, la formation lourde des professeurs (et même une formation légère par correspondance). Le modèle d'alors est la machine à enseigner de Skinner, mais les promoteurs de l'expérience vont avoir un ensemble d'approches géniales pour l'époque : le concept de culture informatique (J. Arsac), la nécessité de portabilité des produits (création du L.S.E., langage symbolique d'enseignement - la suite n'a malheureusement pas confirmé l'option, vraisemblablement par timidité des moyens, manque d'adhésion et querelles d'écoles -, règles d'écriture de logiciels). La formation en Ecole Normale, partie plus tard (début des années 80 avec les premiers micros) prendra appui sur ces conceptions (les premières formations lourdes des professeurs d'Ecole Normale de l'époque seront confiées à l'Université de Jussieu, où enseigne $\mathrm{J}$. Arsac). Le premier degré a depuis gardé son autonomie, sous les influences d'Arsac, de Papert (LOGO), de l'A.F.L. (logiciels ELMO).

En 1981, le rapport Pair-Le Corre avait mis l'accent sur l'informatique au service des disciplines et élément de culture technique.

7 En 1985, le plan Informatique Pour Tous sera l'occasion d'un bilan des acquis, avec sa valise de logiciels complète et un large plan de formation. De fait, en ce qui concerne les enseignements généraux en école et en collège, la valise de logiciels n'a pas depuis pris d'ampleur, sinon par une rénovation des progiciels, surtout le traitement de texte.

1986, critiques officielles du plan I.P.T.; le nouveau plan mettra l'accent sur les simulations et les banques de données, avec le développement de la télématique (dont le volet le plus innovant sera la dotation en serveurs d'établissement). L'abandon de l'édition de logiciels au C.N.D.P. et la politique d'achat de licences mixtes apportera une aide temporaire à l'édition privée, mais ce ballon d'oxygène sera éphémère, car l'initiative est tarie, malgré le lourd investissement en formation réalisé depuis 1985. 


\section{La situation actuelle} magnétoscope, le magnétophone, l'ordinateur, le caméscope, etc., a toujours été à la hauteur du dédain qui n'a pas manqué de suivre à court terme (cf. le thème 5 de l'Inspection Générale, ch. 1). Cela s'est systématiquement traduit par des plans d'équipement mal préparés, sans suivi. Le taux de dysfonctionnement du matériel I.P.T. a dissuadé de nombreuses bonnes volontés d'enseignants déjà réticents à la technique. La mauvaise qualité des outils de reproduction (ajoutée à une mauvaise acoustique des salles de classe) est souvent rédhibitoire (imaginons l'exemple de l'enseignement des langues). Il n'existe pas dans les établissements de personnels d'aide technique, d'où des manutentions très dissuasives (avec en plus la crainte permanente de retrouver le matériel en panne). conservatisme. Cette valeur assez mal portée dans les salles des professeurs sera le plus souvent masquée derrière des considérations plus idéalistes : l'élève a besoin de relation directe avec le maître, dont le rayonnement, le charisme sont d'autant plus nécessaires 
que l'élève est en difficulté ; il faudrait pour faire des outils plus adaptés, des moyens... Au point que l'on a pu faire cesser sans discernement, par exemple la production de logiciels en 1986 sans provoquer la moindre émotion.

Il serait cependant tout à fait injuste de voir dans cette politique chaotique uniquement les comportements velléitaires des décideurs à la fois dans les plans de développement (équipements formation,...) que dans les objectifs. Si l'on a l'impression qu'il n'y a " pas de pilote dans l'avion", ou de "capitaine dans le navire " (vox populi citée dans le rapport Grandbastien), il faut bien dire aussi que la tâche de celui-ci aurait été pour le moins ardue dans son pilotage vers le moyen terme. La prospective sur l'utilisation des technologies nouvelles par l'éducation et le grand public a connu les mêmes errements dans l'ensemble des secteurs (usage culturel de la télévision, plan câble, télématique, informatique domestique, etc.).

19 Les projections dans le futur sont pour le moins aléatoires : il n'est qu'à considérer, pour les années 80 le décalage entre le présent et le futur annoncé ; et les effets d'annonce qui ont tendance à s'amplifier, au rythme des batailles de la guerre économique qui se livrent dans ce secteur, n'éclaircissent pas la situation.

\section{Absence de capitalisation}

Si la France a eu le mérite de défendre ses grandes réussites technologiques comme l'aéronautique, le rail, et les télécommunications..., elle a singulièrement manqué de constance en matière d'éducation. Il existe dans le pays un important vivier de compétences en informatique et audiovisuel. Le rapport Duhamel sur l'enseignement à distance a fait porter les regards vers l'Open Université outre-Manche, modèle «lourd, non transposable, dépassé ", alors que nos atouts et notre potentiel de savoir faire n'ont rien à envier à l'O.U. dans les développements actuels (informatique et télématique). Les critiques du plan I.P.T., en 1986 ont considéré que les choix des matériels étaient plus guidés par le souci d'appuyer la production française que de doter les établissements d'outils performants; en fait quels qu'aient été les choix d'alors, dans tous les cas de figure, les matériels existant à l'époque seraient actuellement obsolètes; pourtant, les nano réseaux seront réformés alors que les développements logiciels n'ont jamais atteint leurs limites de capacité; et les performances (à la fiabilité près) des matériels n'ont jamais bridé les initiatives pédagogiques. Il s'est dispensé une énergie importante dans la réalisation de produits, mais faute d'une organisation (ateliers logiciels), et de normes (norme de portabilité, système documentaire), la majorité d'entre eux n'a pas été maintenue, et un grand nombre est perdu. Il est pourtant envisageable d'effectuer des recensements, des mises à jour et des développements de portabilité, tout en relançant la production.

\section{Absence d'évaluation}

Le nouveau est aussi suspect qu'il est séduisant, et l'on n'évite pas l'éternelle interpellation sur l'évaluation. On se demande ce qu'il adviendrait des outils de communication de la pédagogie traditionnelle : technologie estrade-tableau noir-craie + stylo-cahier, s'ils étaient soumis à une telle sollicitude. Remarquons simplement d'une part que l'évaluation - à supposer que l'on s'entende sur les objectifs, par exemple l'acquisition des programmes scolaires, ce qui reste discutable - ne peut se faire que par rapport aux moyen et long termes (le coût, par exemple, doit être considéré en tenant 
compte de la capitalisation permise par les N.T.I.C., donc des possibilités d'enrichissement et d'évolutivité), d'autant que l'on a peu de chances de faire apparaître un effet transformationnel sur un système en lui appliquant des actions à doses homéopathiques sur des durées courtes (il n'est qu'à considérer les coûts des opérations ci-dessus citées au regard des coûts de l'éducation en France pour se convaincre de la timidité de ces opérations).

L'expérimentation étant l'œuvre de spécialistes ou d'enseignants spécialisés, elle n'a pas toujours bénéficié de l'objectivité nécessaire ; en revanche, les dispositifs techniques ont souvent été vus par les spécialistes des sciences de l'éducation (qui n'ont des techniques qu'une connaissance de deuxième ou troisième main, en général dépassée) comme des outils de médiatisation et non comme médiateurs interactifs, ou vecteurs de communication. Par ailleurs, le temps de la Sociologie et des Sciences de l'Education s'adapte mal au temps de l'évolution technologique et les résultats d'expérimentations sont en général périmés dès leur publication.

Quoi qu'il en soit, les assertions relatives à l'échec des nouvelles technologies sont inexactes. Malgré les difficultés techniques et le manque de suivi des grands projets, un mouvement de fond est en cours, qui ne fera que s'amplifier. La seule question est celle du rythme de son évolution.

\section{S'écarter de la dérive techniciste}

La promotion des N.T.I.C. a donc été laissée, en l'absence de l'indispensable commande des pouvoirs publics, à l'initiative des pionniers, avec un investissement considérable, ses espoirs, ses découragements, et parfois ses travers individualistes. L'éducation, sauf peutêtre l'enseignement technique, n'est pas un lieu de valorisation du progrès. Les méthodes de l'enseignement supérieur sont franchement conservatrices. L'enseignement des sciences en lycée est basé sur la pérennité du concept.

Les N.T.I.C. se trouvent donc face à une nécessité de convaincre à la fois les décideurs politiques et les utilisateurs, non plus dans la perspective de "coups", mais d'une banalisation d'équipement et d'usage. Il est, par conséquent, clair qu'une réflexion prospective ne peut plus, en aucune manière, faire l'impasse sur une réflexion de fond sur les apports psychologiques et psychopédagogiques des technologies d'information et de communication. Des données théoriques sont indispensables pour sortir les Techniques d'Information et de Communication de la dérive techniciste dans laquelle elles sont enfermées, ce qui en fait un terrain facile pour les modes et les affichages politiques. Il est donc urgent de reprendre les choses dans le bon ordre et partir des besoins réels du système éducatif.

\section{N.T.I.C. ou T.I.C.D.}

Le vocable "nouvelles" qui qualifie les techniques actuelles de communication et d'information semble accréditer l'idée de mode. C'est regrettable car cela crée un amalgame entre des techniques éprouvées, telles que le documentaire animé, le montage diapos, ou le rétroprojecteur et des sujets de prospective, comme nous en offrent actuellement la numérisation d'image (D.V.I., par exemple) ou la télématique (réseaux rapides). Dans la suite, sauf pour traiter des techniques réellement nouvelles, nous utiliserons les initiales T.I.C.D., techniques d'information, de communication, et de 
documentation, marquant à la fois le rejet de ces idées de fuite en avant, et notre intérêt pour la documentation, qui est un concept fondamental.

\section{A la recherche de bases épistémologiques et psychologiques}

\section{Les facteurs de l'apprentissage et du développement intellectuel}

\section{Facteurs sociaux}

L'apprentissage est un acte social. «La vie sociale transforme l'intelligence par le triple intermédiaire du langage (signes), du contenu des échanges (valeurs intellectuelles) et de règles imposées à la logique (normes collectives logiques ou prélogiques)» (Piaget, Psychologie de l'intelligence, p. 186). L'accès à la connaissance est une démarche d'intégration à une organisation sociale en évolution, pour des raisons économiques et sociales. Le moteur de l'apprentissage est une volonté d'intégration sociale, le processus s'appuie sur le sentiment de participer à un mouvement d'ensemble social. Le produit du travail est valorisé par son acceptation par le groupe, véhiculé par les représentations, supports de pensée et vecteurs de communication; «la société transforme l'individu en sa structure même, parce qu'elle ne le contraint pas seulement à reconnaître des faits, mais elle lui fournit un système tout construit de signes, qui modifient sa pensée» (Piaget, Psychologie de l'intelligence, p. 186). Sans avoir le monopole des situations de communication authentique à l'école, (les méthodes d'éducation active ont ouvert la voie, il y a plus de cinquante ans), les T.I.C.D. en multiplient la puissance, l'intérêt et la variété. La maîtrise des outils techniques de l'audiovisuel et de l'informatique est, de plus, enjeu de pouvoir. Cela est fortement ressenti par l'enfant et l'adolescent, pour qui les appareils exercent une réelle fascination qui n'est pas encore inhibée par la crainte d'abîmer ou de paraître ridicule; l'enfant peut laisser libre cours à des essais et tâtonnements. Fascination de dominer l'image, à la mesure du rayonnement de la télévision devant laquelle l'enfant passe le plus clair de son temps, mais aussi fascination de dominer le savoir, exemple du plaisir à cheminer dans un CD-Rom.

Le message audiovisuel est de moins en moins témoignage mais de plus en plus expression, il comporte donc une charge affective et sa lecture implique une démarche de communication, cela devient de plus en plus perceptible en informatique et télématique, avec les progrès actuels en matière de convivialité.

Le recours aux TICD, enfin, dédramatisent la métacommunication pédagogique, à savoir la réflexion de l'apprenant sur les modalités de l'apprentissage, tout en donnant lieu à un réel travail en groupes.

\section{Apprentissage et intelligence}

Pour Piaget, la connaissance ne se réduit pas à une mise en mémoire de connaissances, mais elle se construit à partir de l'activité du sujet par un mécanisme d'intégration de nouveaux savoirs (l'accommodation), puis l'équilibration de ceux-ci avec les systèmes de connaissances précédemment acquis (l'assimilation). La prise de conscience (état de savoir) est donc toujours un processus de genèse et non de prise en compte de l'existant dans des structures mentales préétablies. Dans la prise de conscience, l'évolution 
cognitive d'un niveau au niveau supérieur se fait en prenant ce dernier comme objet de réflexion. Cette stratégie permet au sujet de passer du savoir sensible (la perception) au savoir de second degré (le sens) puis au troisième degré (le discours sur la structure ou le concept). L'abstraction se construit par emboîtement : abstraction empirique (portant sur les objets physiques extérieurs au sujet), réfléchissante (procédant des actions et des opérations du sujet), puis réfléchie (désignant la thématisation de ce qui restait opérationnel ou instrumental pour l'abstraction réfléchissante). L'apprentissage est un processus très complexe mettant en jeu différents niveaux de représentation.

31 Pour La Garanderie, "l'introspection éidétique est mise en cuvre pour procéder à l'analyse descriptive des vécus de conscience des concepts fondamentaux de la psychologie cognitive: l'attention, la mémorisation, la compréhension, la réflexion, l'imagination créatrice, pour en dégager les structures opératoires qui les constituent intrinsèquement. La pédagogie y puisera les bases de son action, de telle sorte qu'elle apportera une réponse adéquate à l'exigence de donner aux élèves les moyens d'apprendre à apprendre. » (p.15). L'organisation des savoirs repose sur la composition des évocations, donc des images mentales. La pédagogie procède donc de la mise en œuvre de ces évocations et de la construction du pouvoir symboliser.

L'apprentissage est basé sur l'activité du sujet apprenant. On a ci-dessus mis l'accent sur le rôle privilégié que vont jouer les représentations imagées, sonores, textuelles, non pas en tant que représentation de la réalité (qui n'est pas génératrice d'action de la part du sujet), mais en tant que discours sur la réalité, dans ses déclinaisons de son, d'image fixe ou animée, puis d'image interactive, y compris dans ses représentations textuelles (écran d'ordinateur), puis de discours sur le discours. La médiation simule la construction intellectuelle, en une dialectique d'appel aux images mentales et d'actions du sujet sur ces images mentales, "Le support informatique offre de telles possibilités de matérialisation transitoire de configurations. Il permet d'étendre la fonction de visualisation en lui donnant un substrat perceptif, qui libère momentanément le processeur humain et lui permet d'exécuter sur la configuration présente les opérations cognitives qui pouvaient être exécutées sur l'image. Mais surtout, (...) l'ordinateur est à même de donner un caractère évolutif à cette trace ", " une fonction majeure de l'image est de figurer des hypothèses, d'anticiper mentalement les issues possibles d'un acte créateur. L'image est donc utilisée, non plus pour sa valeur évocatrice d'événements passés, mais dans le contexte d'une préparation d'action » (M. Denis, p. 252).

L'informatique et l'audiovisuel sont des domaines d'exercice privilégié de ce jeu intellectuel avec l'analyse, les scenarii, l'algorithmique, la modélisation, la conduite de l'action.

On conçoit donc la contribution essentielle des T.I.C.D. dans la constitution dynamique des représentations, ainsi que dans la gradation des différents niveaux d'abstraction de représentations, à l'image des «Boucles Etranges ou Hiérarchies Enchevêtrées» de D. Hofstadter.

On sait aussi depuis longtemps que la redondance des supports est un facteur favorable à l'efficacité des apprentissages. Un message oral est mieux appréhendé et retenu s'il est appuyé par du texte et de l'image. Les sujets composant eux-mêmes leurs images mentales à partir des différents supports. L'étude des représentations reste un domaine de recherche active de la psychologie cognitive, et l'on peut s'attendre à une avancée notable des liaisons avec l'intelligence artificielle. Il n'est donc pas déraisonnable d'espérer pour un avenir proche des développements conceptuels relatifs à la syntaxe et à la sémantique du multimédia (et de l'hypermédia). 


\section{T.I.C.D. et pédagogie}

On vient de le voir, connaissance et genèse de l'intelligence procèdent de la réalisation de situations de communication: constitution d'images mentales, capacité à évaluer des situations sur ces images, modélisation et structures, le tout s'appuyant sur le langage et la description des modèles.

7 Le cours traditionnel du maître consiste, dans un dialogue avec un groupe d'élèves, à simuler, par l'échange, un processus de questionnement, de recherche et d'évaluation de solutions, d'exemples et de champs d'application, d'analogies et de différences, de formulations de jugements. Dans les bons cas, la maïeutique est une reconstruction du processus intellectuel.

Les premiers logiciels et les premiers films didactiques ont été construits sur ces modèles en parallèle avec les logiciels d'apprentissage d'inspiration skinnerienne. Les nouvelles générations de logiciels et de vidéogrammes se sont plus ciblés sur des niveaux d'objectifs particuliers. Nous en envisagerons les développements sur les trois axes: supports de cours, banques de ressources dans l'établissement vu comme système d'information, autoapprentissages.

\section{Rénovation de la médiation pédagogique}

39 L'évolution récente des matériels et logiciels ouvre des perspectives très riches dans les supports de cours. Nous pouvons confronter l'élève à des représentations sur écran cathodique, en classe, en appui à la réflexion en commun, soit sous forme de vidéogrammes (non interactifs), de banques d'images ou de programmes informatiques (tous deux interactifs). Je citerai, par exemple les vidéogrammes «formation des montagnes », réalisé par le C.R.D.P. de Montpellier, le vidéodisque « respiration », le CDRom «Megastat", qui modèle les tableaux statistiques en plusieurs dimensions, les travaux faits dans l'Académie de Montpellier sur l'enseignement de la physique et les dispositifs de saisie permettant la visualisation quasi immédiate d'un ensemble de mesures, le logiciel CARTO de géographie, le logiciel « Dérive » en mathématiques, etc. Imaginons aussi la correction ou l'enrichissement d'un texte en commun sur traitement de texte, ou la réflexion sur une série statistique sur un tableur-grapheur.

Ces représentations n'ont pas vocation à remplacer le discours pédagogique, mais sont la simulation d'un phénomène que le professeur peut commenter, avec arrêt sur image, retour,.... L'élève pourra retrouver le document au Centre de Documentation et refaire l'expérience à son rythme pour son propre compte (voir ci-dessous).

Remarquons que dans les exemples ci-dessus, les représentations vont de la représentation du réel (en sciences, en géographie), à la représentation symbolique (cartes de géographie), aux représentations formelles (textes écrits, tableaux numériques, graphiques, formules mathématiques). 


\section{L'établissement vu comme un système de communication} ce câblage multiservice d'établissements (dans l'Académie de Montpellier, les collèges de Quissac, Sommières, Lézignan-Corbières) ont soulevé des problèmes techniques et pédagogiques. Elles ouvrent cependant un champ d'investigations prometteur :

- un véritable centre de ressources. Il est essentiel que les ressources pédagogiques utilisées comme supports de cours (voir ci-dessus) puissent être accessibles en travail autonome de l'élève,

- une communication ouverte sur l'extérieur par la télématique (les élèves participent à la rédaction du service),

- un accès aux ressources externes : banques de données ou messageries externes, émissions étrangères pour l'enseignement des langues,...

- une liaison entre le pédagogique et l'administration-gestion,

- la possibilité de communications entre groupes par circuit fermé de TV,

- la gestion rationnelle des ressources, du point de vue de leur catalogage, la communication sur les ressources, leur protection,...

\section{L'autoapprentissage}

Le travail individuel de l'élève est programmé par le professeur. Il s'agit d'une batterie d'exercices pour renforcer une connaissance opératoire, d'une application, d'une rédaction, dissertation ou résolution de problème, donc, en général, une production plutôt convergente laissant peu de place à l'initiative de l'élève au regard des moyens et méthodes. Il n'en est que plus difficile d'adapter le niveau et la nature des travaux 
personnels demandés à la démarche de l'élève, d'où difficultés d'assimilation, incapacités d'équilibration progressive des connaissances et surtout incapacité de transfert. Sauf à multiplier de façon significative le nombre d'enseignants, on voit difficilement comment atteindre cette individualisation dans le modèle traditionnel. Le recours au livre et au manuel a montré ses limites pour la majorité des élèves (même si l'écrit reste indispensable). En effet, la capacité à l'autocommunication face au texte écrit suppose une dynamique intellectuelle élitiste. On peut voir dans les T.I.C.D. les moyens de compensation, par leur possibilité accrue de générer une action intériorisée, permettre les essais et erreurs, modéliser les images mentales. Cela suppose que des conditions soient réunies. D'abord, il ne saurait y avoir distorsion entre l'enseignement en groupe classe et l'autoapprentissage, ce sont deux démarches totalement imbriquées. Ensuite, il faut que les objectifs de l'apprentissage soient explicites et partagés. L'élève doit avoir été rendu capable d'analyser ses propres succès et échecs (dès le cours préparatoire), la plus grande cause d'échec est la non compréhension de la consigne. Enfin, il faut que le projet d'établissement soit très ambitieux en matière de ressources. Imaginons que l'on croise, mettons pour un collège, l'ensemble des niveaux, l'ensemble des disciplines, la variété des rythmes d'apprentissages, la nécessité de multiplier les niveaux de représentations (tant dans leur niveau de symbolisation: de la copie du réel à la formalisation, que par la multiplicité des supports, image, son, texte,...) et les niveaux d'objectifs (de la connaissance à la résolution de problèmes), cela peut donner une idée d'une masse critique et de l'insuffisance actuelle des produits, en quantité et en qualité. Enfin, il importe qu'un réel système documentaire soit élaboré au service des établissements: recherche intelligente assistée par ordinateur, thesaurus adapté.

\section{Conclusion}

48 "C'est donc bien un génie des procédés éducatifs que nous devons nous attacher à créer. La psychologie cognitive, les sciences de l'éducation, l'économie et la sociologie de la formation, les didactiques des disciplines doivent y contribuer ; les nouvelles technologies de l'information et de la communication sont appelées à y jouer un rôle prépondérant » (M. Grandbastien).

L'ingénierie est aussi un concept qui nous vient de l'entreprise. Elle renvoie aux idées de projet et d'interdisciplinarité. En tant que coordination de spécialités, la pédagogie est concernée au premier chef. Les connaissances sur laquelle cette dernière s'appuie relèvent de psychologie (des psychologies), des sciences de l'éducation. Nous pourrions rechercher ce "génie pédagogique » dans l'agencement dans un ensemble des actions pédagogiques selon leurs niveaux: de médiation, d'objectifs pédagogiques, de communication, son adaptation à des situations et une catégorisation des outils de la pédagogie. Ensemble de processus et de méthodes, l'ingénierie n'est pas un discours politique sur l'école, elle limite son ambition aux concepts relatifs à la conduite de projet, elle fait référence donc en particulier aux notions de définition d'objectifs, de multipartenariat, d'organisation, d'évaluation, de conception de systèmes, d'articulation de modules, de gestion d'itinéraires, de développements de matériels, de veille technologique, etc. Le principe de base de l'ingénierie est la réussite, elle est donc centrée sur le sujet apprenant. Il s'agit en premier lieu d'être en cohérence avec la loi d'orientation, mais surtout prendre acte du fait que le projet d'établissement s'attache à adapter l'enseignement à l'élève, et non, à l'inverse faire passer l'élève au moule d'un 
cursus prédéfini. L'ingénierie pédagogique n'est pas, enfin, recueil de techniques ou de recettes.

Le rôle de l'institution est de fournir, de créer un dispositif d'aide au développement. Celui de la recherche est d'en définir la légitimité épistémologique. La psychologie cognitive, la veille technologique, les recherches actuelles sur la communication, l'image animée, sa syntaxe et sa sémantique, l'intelligence artificielle devront en fournir les éléments théoriques.

BIBLIOGRAPHIE

\section{Rapports :}

NORA S., MINC A., Rapport sur l'informatisation de la Société. La Documentation Française, Paris, 1978.

SIMON J.C., L'Education et l'informatisation de la Société, rapport au Président de la République, Fayard, 1981.

LE CORRE Y., PAIR C., Introduction de l'Informatique à l'Education Nationale, 1981, Service d'Information du Ministère.

CHATEAU J.Y., Les pratiques pédagogiques liées à l'informatique à l'école élémentaire, 1988.

POMONTI J., MICHEL A., Education et télévision, enjeu majeur du XXI ${ }^{\mathrm{ème}}$ Siècle, 1989.

GRANDBASTIEN M., Les technologies dans l'enseignement technique : situation au terme de années 80 et propositions d'orientation pour la décennie à venir, 1989.

BON A., KÜHN M., Médias, multi-médias et télévision au service des formations techniques et professionnelles, 1989

DUHAMEL O., Pour une Université Ouverte, 1990.

DIEUZEIDE H. (col.), Thème 5 de l'Inspection Générale : le rôle des technologies nouvelles (audivisuel et informatique) pour l'aide des élèves en difficulté, 1990.

\section{Ouvrages :}

ARSAC J., La Science Informatique, Dunod, 1970.

LINARD M., Des Machines et des hommes, apprendre avec les nouvelles technologies, collection « Savoir et formation », Editions Universitaires, 1990.

DENIS M., Image et cognition, PUF, 1989.

PIAGET J., (1) La psychologie de l'intelligence, A. Colin, 1947

(2) La prise de conscience, PUF, 1974. 
LA GARANDERIE A. (De), Pour une pédagogie de l'intelligence, Centurion, 1990. HOFSTADTER D., Gödel, Escher, Bach. Interéditions, 1985.

\section{RÉSUMÉS}

Les techniques d'information, de communication et de documentation sont incontournables dans une perspective d'amélioration générale du système éducatif. Leur développement peut s'appuyer sur une masse d'initiatives du terrain, et doit se construire sur des bases épistémologiques, psychologiques, et méthodologiques sûres. C'est le sens du pari de la construction d'une ingénierie des procédés éducatifs.

In order to improve the educational system, the use of information, communication and documentation techniques bas become of utmost importance. Their development is based on initiatives on the field, as well as on epistemological, psychological and methodological grounds. This is the challenge inherent to the study and development of projects involving the educational procedures.

\section{AUTEUR}

\section{EMILE GASPARI}

Directeur du CRDP de Montpellier 